\title{
A Semantically-Adaptive Strategy for Energy- Efficiency in Wireless Medical Monitoring Devices
}

\author{
Vishwa Goudar \\ Computer Science Department \\ University of California, Los Angeles
}

\author{
Miodrag Potkonjak \\ Computer Science Department \\ University of California, Los Angeles
}

\begin{abstract}
We propose a novel strategy for energy-efficient operation of wireless monitoring devices under the premise that medical experts are primarily interested in atypical observations - For epilepsy monitoring, EEG data is most valuable at epileptic activity onset. Or, a gait-stability monitoring application is most interested in unusual footsteps. Observations are atypical if application-specific medical metrics and biosignal features are statistical outliers. Our strategy admits energyefficient early-detection of such observations, leading to: (i) an increase in medical information quality by sampling aggressively over semantically important behaviors, and (ii) a savings in energy by precluding communication of typical measurements. From experimentally collected plantar pressure datasets, we show that this can yield up to a $62 \%$ improvement in gait-stability metric evaluation for atypical footsteps and a $10 \%$ energy cost reduction compared to a recently proposed non-adaptive compressive sensing technique.
\end{abstract}

\section{INTRODUCTION}

While wireless medical monitoring promises vast improvements in applicability and quality of healthcare afforded via mobile patient monitoring systems, powerconsumption and communication bandwidth requirements of such systems pose challenges to large-scale and long term deployments [1] [2].

Fig. 1 illustrates a common Body Area Network (BAN) configuration used in medical monitoring applications. It is comprised of one or more sensors that are capable of extracting important features of the sensed biosignal and transmitting them wirelessly to a base station, such as a smartphone. At the base station, medical metrics relevant to the condition being monitored are computed from the biosignal features, and the metrics are forwarded to a data center for logging. The appeal of this architecture is that the sensors apply their computational power to limit the amount of information transmitted to relevant sensed biosignal features to the base station, where the information is further compressed before it is forwarded. Recently, compressive sensing techniques have been proposed that can further improve performance by a significant margin. By carefully limiting the sampled sensors and their sampling times while maximizing the quality of information elicited with respect the medical metrics being monitored, these techniques are able to produce large savings in power and bandwidth consumption while maintaining high fidelity in the medical metrics produced [3][4].

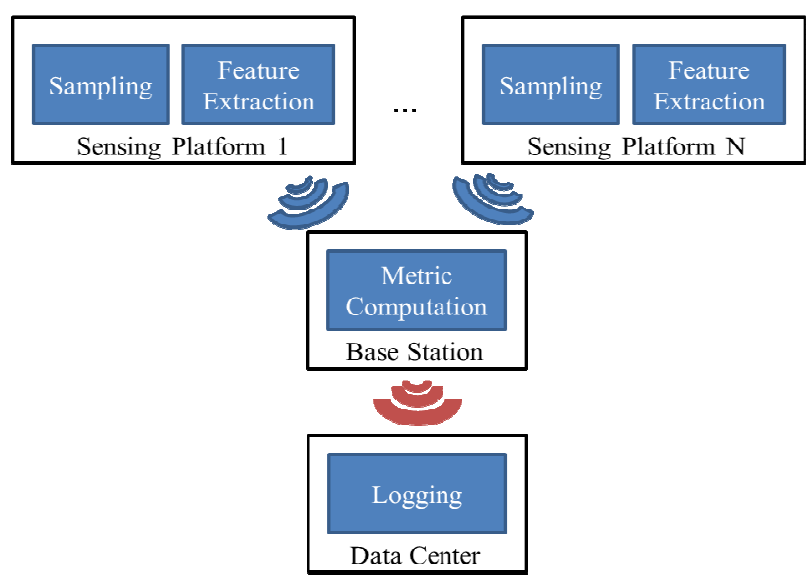

Figure 1. A Common Body Area Network Processing Configuration.

While such techniques correctly characterize the quality of biosignals sensed based on their semantic relevance, the determination of which sensors and sampling epochs produce semantically rich information is often based on statistical techniques and will therefore optimize for the typical case. Rather, we contend that semantically atypical metric observations are much more important to healthcare providers as they may reduce diagnosis times by aiding in early detection of medical conditions. In contrast, techniques that maximize semantic accuracy over typical observations may entirely mis-estimate the metric values during atypical behaviors, delaying diagnosis and making the monitoring device a safety hazard.

In this paper, we present a sampling technique that provides high-fidelity access to semantically atypical metric observations while constraining average energy consumption. Atypical observations are identified by identifying biosignal feature estimates that are likely to yield poor metric estimates, as early as possible. Energy savings arise from identifying typical metric observations as such with fewer biosignal samples at a subset of the available sensors, and precluding transmission of these typical estimates. These savings then offset the higher energy costs arising from increasing the number of sampled sensors and the number of samples taken when the metric is expected to be atypical, thereby producing and transmitting more accurate metric estimates at such times. We validate the proposed method in the context of a multisensor wireless gait stability monitoring system and compare its performance to that in [4] for atypical metric observations. 


\section{RELATED WORK}

A number of signal-processing techniques have crossed over from the domain of Wireless Sensor Network (WSNs) to that of BANs with the goal of reducing power/energy consumption while maintaining signal fidelity. Sensor coverage techniques limit the number of sensors active simultaneously, while ensuring that the targeted area is "covered" without discontinuity. Duty cycling, a form of sensor subsampling, limits the amount of time each sensor node is active, thereby reducing energy consumption. Similar signal processing techniques have crossed over to the domain of BANs [9]. However, these techniques focus on reconstruction and semantic accuracy of an aggregate global signal rather than spatial fidelity of the signal.

Outlier detection techniques have also migrated from WSN to BAN applications, albeit in the context of fault detection. A fault detection technique for BANs was described in [6] wherein the relationship between sensors was modeled to detect outliers. The authors of [7] applied spatial and temporal analyses to BAN sensor samples in order to weed out anomalous measurements and reduce false alarms. In contrast, we apply outlier detection techniques to remedy the shortcomings of energy-efficient signal processing techniques for BANs, by focusing on early detection of statistical anomalies to improve the fidelity of semantic conclusions that may be drawn from the measurements.

\section{USE CASE: Gait Stability MONITORING}

We explore and validate our algorithms in the context of gait stability monitoring. Human gait monitoring enables early detection of several conditions, including stress injuries in runners, plantar ulcers in diabetics and risk of falling in elder populations, to name a few [4]. For example, the average maximum pressure metric has been identified as a causative factor in the development of diabetic plantar ulcers [5]. We explored and validated the performance of our algorithm for this metric based on foot plantar pressure datasets experimentally collected with HERMES, a smart-shoe developed to wirelessly monitor gait stability [8]. It includes 99 passive-resistive pressure sensors located across the shoe insole, that are used to monitor foot plantar pressure as the subject ambulates. The sampling unit for the platform samples each sensor at $60 \mathrm{~Hz}$ with data collected through a 16-bit analog-to-digital converter. Given the reading from HERMES's sensors, the average maximum pressure metric may be measured as the average, over all sensors, of the maximum pressure observed for a stride. The biosignal feature at each sensor is, therefore, the maximum pressure it observes over a footstep.

Based on a dataset of a user's plantar pressure profile collected over several footsteps by HERMES, Fig. 2 illustrates a map of the linear correlation coefficients for the maximum pressure feature between pairs of sensors, where we observe strong linear correlations among groups of sensors. This observation generalized to our other datasets as well. It is this high mutual semantic information among subsets of sensors that is exploited by compressive sensing schemes to limit the number of sensors and samples measured and communicated, while retaining high medical metric fidelity. For this reason,

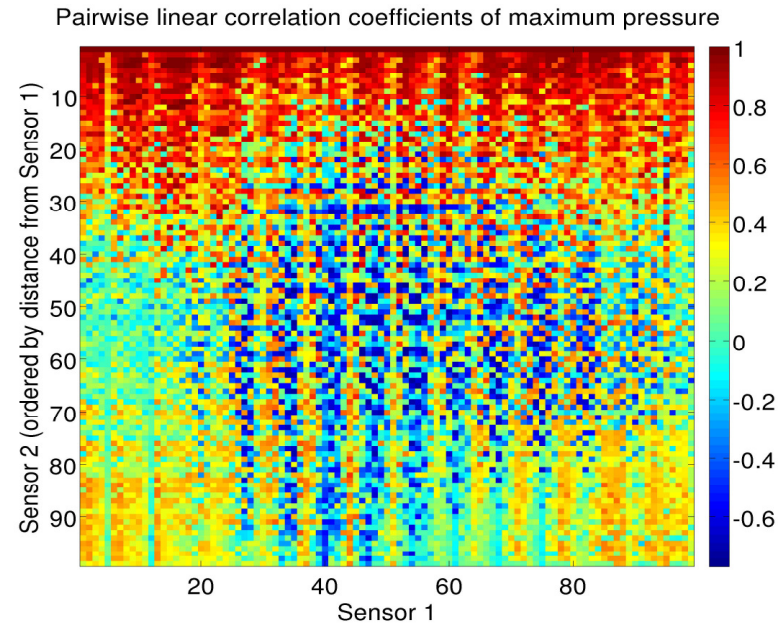

Figure 2. Pair-wise Correlation Map between Sensor Observations of the Max. Pressure Feature over a Foot Plantar Pressure Dataset

our algorithm checks for reductions in the level of mutual information within such subsets to identify user footsteps that appear atypical and are likely to produce low-fidelity medical metric estimates. As discussed, the resolution is to increase the number of active sensors and samples collected to improve metric estimate fidelity.

\section{SAMPLE SELECTION AlgORITHM}

\section{A. Algorithmic Motivation}

In order to predict atypical metric observations in an energy-efficient manner, it is important to do so accurately with few false positives and few false negatives. A large number of false positives will lead to an un-necessary increase in energy usage and many false negatives will lead to loss of information regarding atypical metric measurements. Further, it is also important to produce a detection as early as possible while staying within the energy constraints that apply over typical steps. This translates to selection of samples at each stride such that the energy expended in sampling is within the state constraints and such that the selected samples are able to yield rapid detection.

We identify atypical metric observations as those during which sensors that typically produce observations with high mutual semantic information fail to do so. The reasoning is as follows: With compressive sensing approaches, sensors with high mutual semantic information are unlikely to be sampled together due to the low information gain in sampling one having sampled the other. However, if such sensors begin to produce uncorrelated biosignal feature values, it is likely that the fidelity of the metric estimate, which is based, in part, on the biosignal feature value of the sampled sensor but not the other, will deteriorate owing to a loss in semantic information corresponding to the feature value of the non-sampled sensor. To predict the loss of correlation, our algorithm samples sensors with high mutual information and constructs statistical models to estimate one from the others. If an estimated feature value differs significantly from the actual feature value then an outlier is detected. When the number of detected outliers exceeds a threshold, the metric is expected to be atypical and all sensors are sampled at all the remaining epochs. This, then, 
allows for a more accurate metric estimate to be produced based on the larger number of available samples.

As we have stated, the prediction of atypical metric observations must happen as accurately as possible under constraints on the energy usage, and must happen as early as possible. To fulfill the former requirement, sensors are assigned weights based on how relevant their feature values are to the aggregate metric. Given, a training dataset of typical user footsteps, the feature weight, $f w$, of sensor $s_{i}$, is the coefficient of correlation between $s_{i}$ 's feature value (maximum pressure) and the aggregate metric (average maximum pressure). An inconsistent feature observation at a sensor with a high feature weight translates to a higher likelihood of an atypical metric estimate. Given that the energy constraints are likely to limit the number of sensors that may be sampled, the feature weights will allow the algorithm to prioritize sensors as it constructs the sampling schedule.

To increase the number of sensors that may be sampled under the energy constraints, we limit the number of samples required for a sensor to determine its feature value for each user stride. This will enable sampling of other sensors at other epochs. The training dataset is used to deduce the sampling epochs, featureDistr $\left(s_{i}\right)$, over which $s_{i}$ 's feature value is most likely to be observed and are known as the epochs corresponding to its feature distribution. If scheduled, $s_{i}$ is sampled only at those epochs, and its feature value is deduced from the resulting samples.

To fulfill the second requirement of predicting atypical metric observations as early as possible, the algorithm sections the sampling epochs over a stride into regions to be evaluated independently. Sensors whose feature distribution epochs lie in the earliest region of a stride are evaluated first, and so on. Although sensors are evaluated in the order of their feature weights, independent evaluation of each section/region allows for lower feature weight sensors to be assigned to the sampling schedule before higher feature weight sensors, if their feature distributions correspond to an earlier section. This tradeoff enables early prediction of atypical metric observations, if the sampled sensors in the earlier sections produce semantic outliers. Sections are computed by clustering sensors based on the timing of the sampling epochs corresponding to their feature distribution. The biomechanical notion of phases of a stride naturally lends itself to the notion of epoch sections over a stride - "loading response" is the phase when the heel touches the ground, "mid-stance" when the foot is grounded, and "terminal stance" when the weight shifts to the ball of the foot and the toes. In accordance, k-means clustering is applied to arrive at the section boundaries, with the cluster size set to 3 .

Given that sampling sections are evaluated individually, there arises a need to apportion the energy constraint among the sections. To achieve this, each section is granted a portion of the energy budget that is proportional to the level of contention observed in the section. The level of contention of each sampling epoch in a section is determined based on the feature weights of sensors vying for that epoch. The ratio between the average contention of the sections determines apportionment of the energy budget. This notion of contention
TABLE I.

CONTENTION-AWARE REGRESSION



is central to the construction of the sampling schedule in the following sense: assignment of a sensor's feature distribution to the sampling schedule reduces the likelihood that another's feature distribution will get assigned (or may preclude it entirely). Therefore, higher contention sections must be budgeted more samples, and consequently more energy.

\section{B. Semantically Adaptive Scheduling Algorithm Description}

The sample schedule construction algorithm proceeds as follows. Sections are evaluated in sequence from earliest to latest. Over each section, sensors whose feature distributions the section includes are evaluated in the order of their feature weights. The assignment of a sensor, $s_{i}$, subsumes the assignment of the other sensors $s_{k}$, whose feature values will be used to estimate that of $s_{i}$. The set of sensors, $s_{k}$, for a given $s_{i}$ is computed based both on their ability to predict $s_{i}$ 's feature value well, and on the increase in contention and assignment preclusion, if any, that their assignment results in. The algorithm moves on to the next section, either when all sensors corresponding to the current section have been assigned, or if the remaining energy budget for the section nolonger allows the assignment and estimation of any sensor's feature values.

Finally, Table 1 outlines the regression algorithm used to arrive at the set of sensors that will be used to estimate the currently evaluated $s_{i}$ 's feature value. At each iteration, the algorithm evaluates all sensors available for estimation and assigns a cost to their assignment. The sensor with the smallest cost is then selected for assignment at the end of the iteration (lines 14-17). To enable early prediction, the 


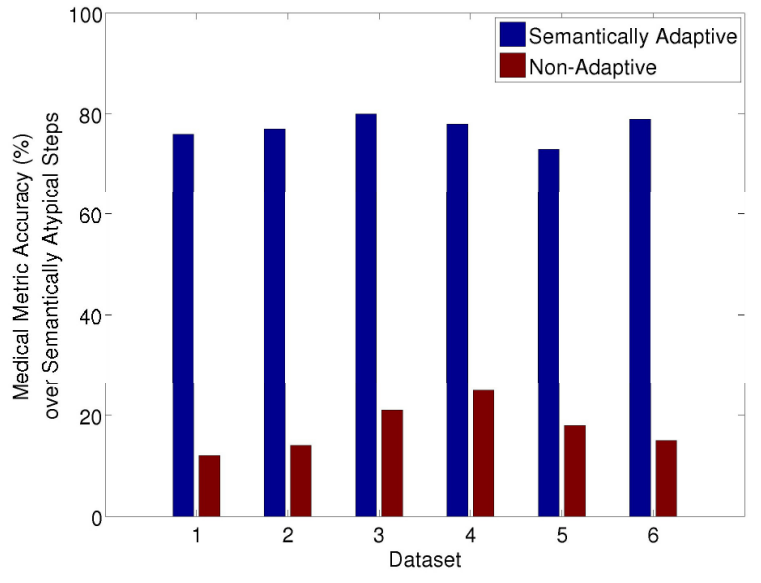

Figure 3. Accuracy of Average Max. Pressure Metric estimates, averaged over semantically atypical steps in each dataset, with and without the proposed adaptive sampling algorithm.

algorithm estimates $s_{i}$ 's feature values based only on that of sensors whose featureDistr occur at or before it (line 4). Further, a sensor is only considered as an independent variable for the estimation if it contributes to the improvement of the coefficient of determination $\left(R \_s q\right)$ of the estimate by a minimum threshold value $\varepsilon$ (lines 6-8). This enables the production of robust regression estimates. The cost of using a sensor $s_{j}$ that passes this test is based on the extent to which it improves the estimate of a sensor such as $s_{i}$ with feature weight $f w\left(s_{i}\right)$ and the extent to which it will help estimate the feature values at other sensors that have not been estimated thus far. It is also penalized for the extent to which it increases contention, or precludes other assignments (line 11). Finally, the algorithm returns with the assignments made, once a minimum requisite coefficient of determination has been achieved by the estimate (lines 20-22). No assignments are made if this quality of estimation cannot be achieved (line 16), or if the energy budget has been depleted (lines 23-25).

\section{RESULTS}

We validate our adaptive algorithm on six experimentally collected datasets of foot plantar pressure measurements, each comprising of a few hundred footsteps. The datasets correspond to different subjects and were collected with the HERMES platform. Each dataset was divided into a training subset comprised of $80 \%$ of the footsteps, all of them typical, with the remaining $20 \%$ used for testing. Half of testing subset consisted of typical steps, where the subject walked forward normally, and the other half of atypical steps where the subject shuffled her/his feet. We compared performance to the compressive sensing technique described in [4]. To keep the results comparable, the energy budgets for our algorithm were based on a maximum number of samples given by 5 times the number of sampling epochs over a footstep.

Fig. 3 compares the metric accuracy of our adaptive algorithm when the threshold number of outlier detections required to categorize a step as atypical is set to 2 , to the metric accuracy in [4] under similar energy constraints. Clearly, our proposed strategy produces substantially more accurate metric estimates for atypical footsteps over 6 all datasets (increase of between $57 \%$ and $62 \%$ in prediction



Figure 4. Average change in energy costs and metric accuracy for semantically atypical steps, as the number of outlier detections necessary to classify a step as atypical is changed. Values are averaged over all datasets.

accuracy). The error that remains, arises from the delay in categorizing the step as semantically atypical. Fig. 4 compares the accuracy that can be achieved for atypical steps to the change in energy costs, which arise when the threshold number of outlier detections is varied. Here, the average energy costs over the testing set increased due to sampling and transmission over atypical steps and a similar increase over mis-classified typical steps, and decreased due to nontransmission over correctly classified typical steps. Depending on the desired increase in accuracy, a net-decrease of up to $10 \%$ was achievable in comparison to an already efficient compressive sensing algorithm while still achieving more accurate metric estimates.

\section{REFERENCES}

[1] P. Pawar, V. Jones, B. F. van Beijnum and H. Hermens, "A framework for the comparison of mobile patient monitoring systems", In J. Biomed. Informatics, vol.45, no.3, 2012, pp. 544-556.

[2] M.A. Hanson, H.C. Powell, A.T. Barth, K. Ringgenberg, B.H. Calhoun, J.H. Aylor and J. Lach, "Body Area Sensor Networks: Challenges and Opportunities", In Computer vol.42, no.1, 2009, pp. 58-65.

[3] J. B. Wendt, S. Meguerdichian, H. Noshadi, and M. Potkonjak, "Semantics-driven Sensor Configuration for Energy Reduction in Medical Sensor Networks," In Proc. ACM/IEEE Inter. Symp. on Low Power Electrn. and Design, 2012, pp.303-308.

[4] V. Goudar and M. Potkonjak, "Power constrained sensor sample selection for improved form factor and lifetime in localized BANs", In Proc. Conf. on Wireless Health, 2012.

[5] P.R. Cavanagh and S.A. Bus, "Off-loading the diabetic foot for ulcer prevention and healing,” In JAPMA, vol.100, no.5, 2010, pp.360-368.

[6] D. Kim and B. Prabhakaran, "Motion fault detection and isolation in Body Sensor Networks”, In Pervasive Mob. Comput., vol.7, no.6, 2011, pp. 727-745.

[7] O. Salem, Y. Liu and A. Mehaoua, "A lightweight anomaly detection framework for medical wireless sensor networks," In Proc. Wireless Comm. and Net. Conf., 2013, pp.4358-4363.

[8] H. Noshadi H., S. Ahmadian, H. Hagopian, J. Woodbridge, F. Dabiri, N. Amini, et. al., "Hermes - Mobile Balance and Instability Assessment System”, In Proc. Conf. on Bio-Inspired Sys. and Sig., 2010, pp. 264270 .

[9] V. Goudar and M. Potkonjak. "Energy-efficient sampling schedules for body area networks.", In Proc. IEEE Sensors, 2012, pp.1-4. 